\title{
Relaciones de poder en tareas de co-enseñanza: un estudio de casos entre pares de profesores en Chile
}

\author{
Power relations in co-teaching tasks: a case study among pairs of teachers \\ in Chile
}

\section{Relações de poder em tarefas de co-ensino: um estudo de caso entre pares de professores no Chile}

Fabián Andrés Inostroza Inostroza Universidad Católica de la Santísima Concepción, Facultad de Educación, Chile

fainostroza@ucsc.cl http://orcid.org/0000-0003-1530-2266

\section{Resumen}

En Chile, la coenseñanza ha sido planteada como una medida eficaz desde las políticas de inclusión para mejorar la calidad de los aprendizajes de todos los estudiantes, en especial para favorecer los procesos educativos de niños que presentan dificultades de aprendizaje. No obstante, las investigaciones existentes hasta el momento sobre este fenómeno solo se han centrado en describir e identificar barreras y facilitadores en el trabajo docente en conjunto, sin problematizar las relaciones de poder que emergen cuando los educadores laboran en parejas. Por ende, el objetivo de esta investigación es conocer — desde una perspectiva analítica foucaultiana- las relaciones de poder presentes en las tareas de coenseñanza entre dos duplas de profesores en su trabajo pedagógico cotidiano. Metodológicamente, se optó por desarrollar un estudio de casos con enfoque etnográfico para detectar la presencia de relaciones asimétricas de poder relacionadas, en primer lugar, con los saberes o el saber-poder que cada uno de los profesionales ejerce para legitimar su estatus como experto de la enseñanza o como especialista en inclusión y dificultades del aprendizaje. En segundo lugar, se evidencia una disputa por el espacio y la autoridad dentro del aula 


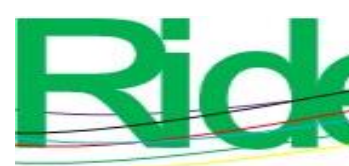

Revista Iberoamericana para la
Investigación y el Desarrollo Educativo
ISSN $2007-7467$

foucaultiana - as relações de poder presentes nas tarefas de co-ensino entre duas duplas de professores em seu cotidiano de trabalho pedagógico. Metodologicamente, optou-se por desenvolver um estudo de caso com abordagem etnográfica para detectar a presença de relações assimétricas de poder relacionadas, em primeiro lugar, ao saber ou saber-poder que cada um dos profissionais exerce para legitimar o seu estatuto de perito em como docente ou como especialista em inclusão e dificuldades de aprendizagem. Em segundo lugar, há uma disputa sobre espaço e autoridade dentro da sala de aula regular. Especificamente, percebeuse um exercício de poder a favor dos professores do ensino fundamental, de forma que educadores diferenciais foram colocados à margem da participação em sala de aula.

Palavras-chave: co-ensino, poder, professores no Chile, relações de poder.

Fecha Recepción: Julio 2020

Fecha Aceptación: Diciembre 2020

\section{Introducción}

Esta investigación tiene como propósito conocer las relaciones de poder presentes en tareas de coenseñanza entre dos parejas de docentes de escuelas subvencionadas de Santiago de Chile. El estudio se realizó siguiendo una tradición cualitativa de investigación en ciencias sociales, y se desarrolló por medio de un diseño de estudio de casos con un enfoque etnográfico, en el que durante una fase exploratoria se aplicaron entrevistas en profundidad a 40 actores educativos (20 educadoras diferenciales y 20 profesores y profesoras de educación regular). Luego, en la etapa denominada profundización, se efectuó observación no participante con una duración de un semestre académico a cada dupla de pedagogos seleccionados como casos. De este modo se compilaron aproximadamente 240 horas de registro a través de notas de campo y 40 entrevistas en total.

Analíticamente, se privilegió el uso de una perspectiva postcrítica para el análisis del corpus discursivo, siguiendo los lineamientos planteados por Foucault (2013) para conocer las relaciones de poder en las tareas de coenseñanza entre dos parejas de docentes, constituida cada dupla por un profesor de educación básica y una educadora diferencial. Este enfoque fue útil en la medida que permitió determinar las diversas formas en las que el poder circulaba y se ejercía en las interacciones en el marco del trabajo pedagógico cotidiano entre educadores. 


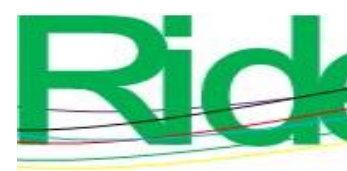

Revista Iberoamericana para la Investigación y el Desarrollo Educativo ISSN 2007 - 7467

La coenseñanza ha sido un fenómeno que desde la perspectiva de la inclusión educativa se ha relevado como una forma de trabajo colaborativo entre docentes, pero principalmente como un medio para lograr una educación más equitativa. Esta tiene la potencialidad de brindar igualdad de oportunidades de aprendizaje para todos los educandos por medio del diseño de una enseñanza ajustada a las diversas necesidades de los alumnos (Friend, 2008).

A nivel internacional se ha detectado que la coenseñanza ha contribuido a la planificación de procesos de enseñanza-aprendizaje entre docentes de educación regular (básica o primaria) y educadores diferenciales o de educación especial (special education teachers), lo que ha favorecido la diversificación de los procesos educativos dirigidos al alumnado con necesidades educativas especiales (NEE, de aquí en adelante), como también en la producción curricular contextualizada y el desarrollo profesional docente continuo (Cook y Friend, 1995). Además, se ha reportado en distintas latitudes del mundo que la coenseñanza, en general, se ha desarrollado por medio de análisis de duplas de coenseñantes, identificando barreras para este trabajo, tales como falta de tiempo, limitada formación inicial del profesorado para el trabajo colaborativo, escasa claridad entre los roles y responsabilidades entre profesores y falta de apoyo por parte de los directivos de los establecimientos escolares (Friend, 2008; Scruggs, Mastropieri y McDuffie, 2007). A su vez, se han evidenciado beneficios de la enseñanza entre dos pedagogos, específicamente en el trabajo interdisciplinario, en la colaboración docente y en la construcción de procesos de enseñanza en conjunto.

Considerando los hallazgos mencionados con anterioridad, en un ámbito relacionado se ha prestado un interés especial en la investigación centrada en la formación inicial y continua de los docentes, dada la relevancia que tiene la preparación de los futuros educadores y profesores que se encuentran ejerciendo para mejorar los procesos de colaboración y trabajo en conjunto (Aliakbari y Bazyar, 2012; Indrisano, Birmingham, Garnick y Maresco, 1999).

En tanto, en Chile, el fenómeno de la coenseñanza ha sido abordado principalmente desde la promulgación del decreto 170 del año 2009, política que buscó promover el trabajo colaborativo como una estrategia para el desarrollo de procesos de enseñanza-aprendizaje diversificados. Entre los estudios existentes, estos dan cuenta de una cultura de trabajo pedagógico caracterizada por el aislamiento entre los docentes (balcanización), como también de incipientes iniciativas de trabajo de coenseñanza entre profesores de aula regular 


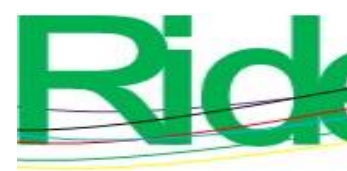

Revista Iberoamericana para la Investigación y el Desarrollo Educativo ISSN $2007-7467$

sujetos que se encuentran en una posición de inferioridad. Más bien, el poder será comprendido como una estrategia, algo que produce y que configura la realidad social y las subjetividades de los sujetos (Grinberg, 2008).

En este sentido, el poder corresponde a "una situación estratégica compleja, como una multiplicidad de relaciones de fuerza (...) simultáneamente intencional y a la vez no subjetivas" (Foucault, 2013, p. 116). Por ello, para el intelectual francés el poder es inmanente a las relaciones sociales, es decir, en donde existan interacciones entre seres humanos existirán relaciones de poder, y este poder circulará, fluirá y será ejercido por los sujetos y al mismo tiempo configurará la realidad en la que estos se encuentran.

Esta forma de concebir al poder lo sitúa en el seno mismo de las relaciones sociales, ya que el poder constituiría a los sujetos y fluiría a través de estos; por ello, esta noción es un mecanismo singular, que está inserto en el tejido social, el que se infiltra en las dimensiones más íntimas y privadas de la vida de los seres humanos, ya que el poder es cosustancial a la sociedad en donde los sujetos adoptan diversos posicionamientos.

Para los fines de esta investigación, esta noción de poder resulta de utilidad, ya que permitirá identificar cómo el poder es ejercido, cómo circula y cómo se distribuye entre los sujetos. En efecto, el poder como una estrategia no se aplica de manera vertical ni de forma vectorial, sino que su funcionamiento se asemeja a campos de fuerza en disputa en donde su distribución depende de la posición de los sujetos y los contextos sociohistóricos en donde se encuentran situados.

Vinculadas con la noción de poder elaborada por Foucault se encuentran las relaciones de saber-poder. El intelectual francés postula que la producción de saberes está asociada directamente con relaciones de poder dentro de una sociedad determinada (Ball, 1997). Es decir, desde este enfoque existen disciplinas científicas como medicina, psiquiatría, derecho, economía, entre otras, cuyos saberes son legitimados y validados respecto a otros, considerándolos como verdades incuestionables (Popkewitz, 2004). Esta legitimación — de acuerdo con Foucault (2006) — se lleva a cabo por medio de determinadas prácticas de poder, que las instituye como la "verdad" en un campo del conocimiento determinado (Ball, 1997).

Un ejemplo de lo anterior, pero extrapolado al ámbito educativo, es que se han identificado ciertas relaciones de saber-poder validadas a nivel de políticas gubernamentales (Ley Subvención Escolar Preferencial, decreto 83, Programa de Integración Escolar o PIE), así como a través de disciplinas que poseen un estatus especial. Por ello, se consideran como discursos "hegemónicos" en educación especial ciencias como la medicina y la psicología, 


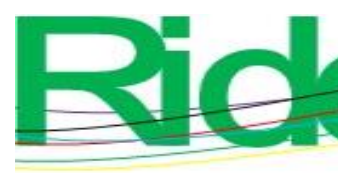

Revista Iberoamericana para la Investigación y el Desarrollo Educativo ISSN $2007-7467$

que son legitimadas para diagnosticar y tratar a los niños con NEE en el marco de un mercado educativo como lo es el chileno (Peña, 2013).

\section{Coenseñanza}

La coenseñanza se enmarca en el estudio del trabajo colaborativo, en el que dos o más docentes se proponen desarrollar procesos de enseñanza-aprendizaje destinados a un grupo determinado de educandos, lo que incluye la planificación, instrucción y evaluación conjunta (Cramer, Liston, Nervin y Thousand, 2010; Rodríguez y Ossa, 2014; Villa, Thousand y Nevin, 2008).

Autores como Conderman y Hedin (2012) han determinado que en este trabajo en conjunto existen al menos tres momentos o componentes en los que se puede comprender de manera más pragmática a la coenseñanza; estos corresponden a coplanificación, coinstrucción y coevaluación.

La coplanificación implica la elaboración del plan de clases entre el profesor de educación básica y la educadora diferencial en un espacio dentro de la escuela habilitado para tales fines; en cambio, la coinstrucción remite a la enseñanza en la sala de clases regular con todo el alumnado, en donde ambos pedagogos impartirán la clase planificada en conjunto. Por último, la coevaluación es el trabajo pedagógico entre ambos pedagogos en donde consensuan las alternativas más óptimas y adecuadas para calificar y valorar los procesos de aprendizaje de los alumnos (Suárez-Díaz, 2016).

En la literatura se han reportado múltiples aportes y beneficios de la coenseñanza, entre estos se incluyen la diversificación de la enseñanza, aumentar la coherencia entre los contenidos que dos o más profesores imparten de una misma asignatura, el desarrollo profesional docente por medio de la reflexión y retroalimentación entre el profesorado, la mejora en la calidad de los aprendizajes adquiridos por los educandos y también ha contribuido en el trabajo cooperativo entre el profesorado (Cook y Friend, 1995; Moliner, 2008; Strogilos y Stefanidis, 2015).

Los estudios relativos a la coenseñanza en el contexto chileno son de reciente data, pues emergen con los lineamientos del decreto 170 del año 2009 del Ministerio de Educación (Mineduc, 2009), donde se prescribe el trabajo pedagógico colaborativo entre profesores de aula regular y educadoras diferenciales con la finalidad de atender las necesidades educativas especiales (NEE) de los educandos que asisten a escuelas que cuentan con programas de integración escolar (PIE). 


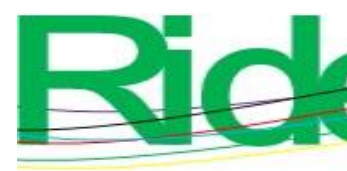

Revista Iberoamericana para la
Investigación y el Desarrollo Educativo
ISSN $2007-7467$

Adicionalmente, la literatura examinada sobre la coenseñanza en escuelas chilenas ha demostrado que existen diversas barreras para su desarrollo, como la balcanización o el aislamiento de los docentes en su trabajo pedagógico cotidiano (Marfán et al., 2013), las distintas políticas que prescriben sus respectivos trabajos pedagógicos (Inostroza, 2020) y la relación solo de "apoyo" - y no de intercanbio mutuo de saberes - entre los docentes de aula regular y de educación especial (Rodríguez y Ossa, 2014).

\section{Metodología}

Esta investigación se basó en una tradición cualitativa (Creswell, 2007; Patton, 1990) que favorece la comprensión en profundidad de un fenómeno social de interés para el investigador. La perspectiva epistémica que orienta a este estudio se sustenta en un enfoque poscrítico enfocado en objetos de estudio relacionados con la (in)justicia, la (in)equidad, las (des)igualdades y las relaciones de poder presentes en una realidad social en un espaciotiempo determinado (Cherryholmes, 1999; Da Silva, 2001).

\section{Diseño}

Este estudio se desarrolló en dos fases: en la primera —denominada exploratoriase realizaron entrevistas en profundidad a 20 educadoras diferenciales y 20 profesores y profesoras de educación básica de la Región Metropolitana, los que fueron seleccionados a través de un muestreo cualitativo intencionado y de acuerdo con su disponibilidad (Cardona, 2002; Tójar, 2006). Las entrevistas permitieron compilar un corpus discursivo amplio que contribuyó a una primera aproximación a los discursos que sostenían los docentes de aula y las maestras de educación especial respecto a las relaciones de poder en las interacciones pedagógicas que sostenían en sus respectivos contextos de desempeño cotidiano.

En la segunda etapa — denominada de profundización — se desarrolló un estudio de casos múltiple (Yin, 2010) a dos parejas de profesionales de la educación, cada dupla conformada por una educadora diferencial y un profesor de educación básica (primaria). A estos participantes se les realizó un seguimiento etnográfico (Guber, 2004; Rockwell, 2009) durante un semestre académico (equivalente a cinco meses), y se les aplicaron entrevistas en profundidad para conocer las relaciones de poder presentes en las interacciones pedagógicas que sostuvieron durante los meses en los que el investigador se encontró realizando el trabajo de campo. 


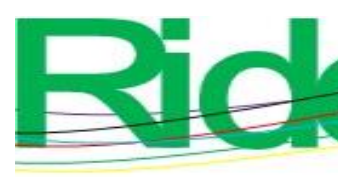

Revista Iberoamericana para la
Investigación y el Desarrollo Educativo ISSN $2007-7467$

\section{Participantes}

Durante la primera etapa se empleó un muestreo cualitativo de tipo intencionado y de acuerdo a con disponibilidad de los participantes (Cardona, 2002; Tojár, 2006). Este contó con la participación de 20 educadoras diferenciales y 20 profesores y profesoras de educación general básica. Como criterios de diferenciación se estimó que los actores educativos pertenecieran a instituciones educativas de distinta dependencia administrativa, de dispares posiciones en el mercado educativo chileno, de diversas provincias de la Región Metropolitana, escuelas que atendían a alumnado con distinto nivel de vulnerabilidad social y con variablidad en su tamaño (entre 300 y 1200 educandos matriculados), los que contaban a su vez con Programas de Integracion Escolar (PIE), es decir, admitían a educandos categorizados con NEE.

En la segunda fase se optó por la selección intencionada y por criterios (Patton, 1990) de dos parejas constituidas cada dupla por una educadora diferencial y un profesor de educación básica. Una de las parejas estuvo conformada por la educadora especial o diferencial —llamada Sofia - y un profesor de educación básica —llamado Raúl—. Ambos pertenecían a una escuela municipal, establecimiento que poseía con un nivel de vulnerabilidad del $98 \%$ del alumnado y localizado en una sector clasificado de vulnerabilidad social en la Región Metropolitana; además, contaba con una matrícula de aproximadamente 800 estudiantes.

Respecto a la otra dupla de profesionales, estaba compuesta por una educadora diferencial que fue nombrada como Patricia y un profesor de educación básica llamado José. Ambos se desempeñaban en un centro escolar particular subvencionado (con subvención estatal y financimiento de los padres) perteneciente a una congregación religiosa católica, que se encontraba emplazado en un sector en donde residían familias de clase media baja, con un nivel de vulnerabilidad del alumnado del $80 \%$ y con un matrícula total del colegio de 1200 alumnos, aproximadamente.

En la tabla 1 se presentan algunas características de los actores educativos correspondientes a los casos de estudio: 


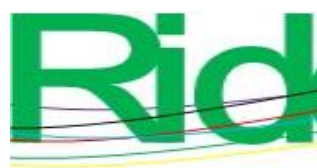

Revista Iberoamericana para la Investigación y el Desarrollo Educativo ISSN $2007-7467$

Tabla 1. Características de las educadoras y profesores pertenecientes a los casos de estudio

\begin{tabular}{|c|c|c|c|c|}
\hline Dupla 1 & $\begin{array}{c}\text { Formación } \\
\text { inicial }\end{array}$ & Edad & Cargo o funciones & $\begin{array}{c}\text { Años de } \\
\text { trayectoria }\end{array}$ \\
\hline Sofía & $\begin{array}{l}\text { Educadora } \\
\text { Diferencial } \\
\text { mención } \\
\text { Discapacidad } \\
\text { Intelectual }\end{array}$ & 36 & $\begin{array}{l}\text { Educadora diferencial } \\
\text { coordinadora de segundo } \\
\text { ciclo }\left(5 .^{\circ} \text { a } 8 .^{\circ} \text { grado) }\right.\end{array}$ & 7 \\
\hline Raúl & $\begin{array}{l}\text { Licenciatura en } \\
\text { Lengua y } \\
\text { Literatura. } \\
\text { Profesor de } \\
\text { Lenguaje y } \\
\text { Comunicación. }\end{array}$ & 60 & $\begin{array}{l}\text { Profesor de Lenguaje y } \\
\text { Comunicación en el } 7 .^{\circ} \mathrm{y} \\
\text { 8. } .^{\circ} \text { grado. }\end{array}$ & 21 \\
\hline \multicolumn{5}{|l|}{ Dupla 2} \\
\hline Patricia & $\begin{array}{l}\text { Educadora } \\
\text { Diferencial } \\
\text { mención } \\
\text { Trastornos del } \\
\text { Aprendizaje }\end{array}$ & 28 & $\begin{array}{l}\text { Educadora Diferencial } \\
\text { encargada del primer } \\
\text { ciclo ( } 1 .^{\circ} \text { a } 4 .^{\circ} \text { grado) }\end{array}$ & 5 \\
\hline José & $\begin{array}{l}\text { Profesor de } \\
\text { Educación } \\
\text { General Básica } \\
\text { con mención en } \\
\text { Matemáticas }\end{array}$ & 48 & $\begin{array}{l}\text { Profesor de matemáticas } \\
\text { entre el } 3 .^{\circ} \text { y } 6 .^{\circ} \text { grado. }\end{array}$ & 15 \\
\hline
\end{tabular}

Fuente: Elaboración propia

\section{Procedimientos}

En la etapa exploratoria se envió una invitación por medio de una carta certificada y los consentimientos informados correspondientes a diversos establecimientos educacionales de distintas provincias de la Región Metropolitana. A la participación en este estudio en esta fase, accedieron 20 educadoras diferenciales y 20 profesores y profesoras de educación básica. A estos actores educativos se les realizó una entrevista en profundidad para indagar en los discursos que estos pedagogos produjeron en torno a las interacciones y relaciones de trabajo que sostuvieron en tareas de coenseñanza en sus respectivos contextos de desempeño. Las entrevistas se realizaron en cada uno de los centros escolares dentro de la jornada escolar de cada profesional de la educación, con una duración de cada entrevista de una hora aproximadamente. Estas fueron grabadas en audio y posteriormente transcriptas, compilando un total de 40 horas de audio. 


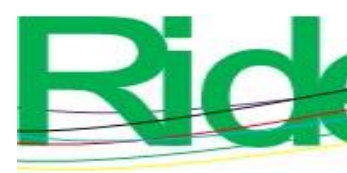

Revista Iberoamericana para la
Investigación y el Desarrollo Educativo
ISSN $2007-7467$

En la fase de profundización se implementó un seguimiento etnográfico a los casos de estudio. Durante el primer semestre del año 2018, se realizaron observación no participante durante una vez a la semana por seis horas cronológicas durante cinco meses; el procedimiento se replicó en la segunda dupla del colegio particular subvencionado en el segundo semestre lectivo del mismo año. De manera paralela, se desarrolló una nueva ronda de entrevistas en profundidad a los actores que constituyeron a cada una de las duplas. Las entrevistas tuvieron una duración de una hora y media de grabación de audio aproximadamente. Ambas técnicas de recolección de información se desarrollaron con la finalidad de conocer con mayor profundidad las diversas maneras en que se desplegaban y ejercían las relaciones de poder en ambas parejas de educadores en sus respectivos contextos de trabajo cotidiano. En total se compilaron 120 horas de observación no participante por dupla, con un total de 240 horas correspondientes a las dos parejas y seis horas de grabación de audio producto de las entrevistas concretadas.

\section{Técnicas de análisis}

Para la sistematización de la información producida en la fase de exploración (entrevistas en profundidad) se procedió en una primera instancia por medio de un proceso de codificación abierta proveniente de la teoría fundamentada (Strauss y Corbin, 1998). Luego se analizaron las categorías que emergieron de un proceso de lecturas iterativas del corpus discursivo a través del análisis de contenido cualitativo (Mayring, 2000) con la utilización de manera instrumental del software de análisis cualitativo Atlas ti $® 8.1$.

Posteriormente, para organizar la información compilada en la fase de profundización, se emplearon las categorías que emergieron en la etapa anterior del análisis. De este modo se sistematizó el corpus proveniente de las notas de campo y de las entrevistas en profundidad a los casos de estudio. Para estos efectos, se desarrolló una estrategia de análisis cruzado, empleando en primer lugar análisis de contenido cualitativo (Mayring, 2000) y en un segundo momento, se conceptualizaron las categorías de análisis que se presentarán a continuación: el estatus del experto y de las especialistas, y la disputa por el espacio y la autoridad en el aula regular. 


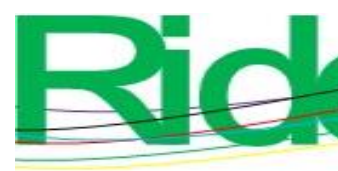

Revista Iberoamericana para la
Investigación y el Desarrollo Educativo ISSN 2007-7467

\section{Resultados}

Entre los hallazgos más relevantes se evidenciaron relaciones de poder asimétricas en cuanto a los saberes específicos que detentaba cada uno de los educadores, relacionados por un lado con su formación inicial y correspondientes especificidades en sus roles (denominada esta categoría como estatus del experto y de las especialistas); igualmente, se identificaron relaciones de disputa por el espacio y el poder dentro de la sala de clases (categorizada como disputa por el espacio y la autoridad en el aula regular).

\section{EI estatus del experto y de las especialistas}

La formación inicial docente en Chile de educadoras diferenciales y de profesores de educación básica (o primaria) difiere en varias dimensiones. Si bien en ambos programas de preparación del profesorado se comparten asignaturas relacionadas con los fundamentos de la educación, el currículo escolar, la evaluación del aprendizaje y la atención a la diversidad, las maestras de educación especial centran su formación en la adquisición de conocimientos y habilidades vinculadas con las dificultades de aprendizaje del alumnado, la evaluación de las NEE, la diversificación curricular, entre otros. En cambio, los profesores y profesoras de educación primaria focalizan su preparación profesional en las didácticas específicas (lenguaje, matemáticas, historia, ciencias, etc.) y en las habilidades básicas para desenvolverse en el aula (por medio de prácticas iniciales, intermedias y profesionales).

Estas diferencias en la formación inicial y en sus identidades docentes se evidenciaron en los discursos que los profesores y educadoras produjeron en la fase exploratoria, ya que se centraron en diferenciar los saberes que cada profesional portaba y hacía circular, y desde dicha posición legitimar o deslegitimar a sus colegas cuando debían referirse a ellos y al trabajo pedagógico que desempeñaban en el cotidiano.

Un ejemplo de ello es el fragmento que se presenta a continuación, emitido por una de las profesoras de educación básica, quien se refirió a los saberes que ellos y ellas detentaban y la distinción que construían discursivamente respecto de las educadoras diferenciales:

Nosotros somos especialistas en enseñar, en la enseñanza de distintas asignaturas, en mi caso de matemáticas, y por lo tanto sabemos de didáctica, de la transposición y de cómo contextualizar las materias. Y eso mismo nos permite enseñar cosas de forma más profunda y a todos los niños, por ejemplo, 


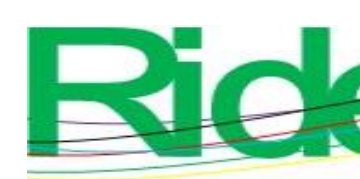

Revista Iberoamericana para la
Investigación y el Desarrollo Educativo
ISSN $2007-7467$

una educadora diferencial no sabe mucho de geometría, álgebra o de potencias. Y eso pasa porque ellas solo trabajan con niños con problemas, en eso ellas son especialistas y su trabajo es como más terapéutico con estos niños (24 de mayo de 2018, entrevista 14).

En este fragmento se aprecia cómo los profesores de educación básica se otorgaban a sí mismos el estatus de "expertos” en la enseñanza; de hecho, se posicionaban como quienes conocen la manera de impartir las diferentes asignaturas en el aula regular a todo el alumnado, y no solo a un grupo de ellos. En este sentido, se atribuían el rol de protagonistas en el proceso de enseñanza-aprendizaje, desplazando a las educadoras diferenciales a otro plano. En concreto, se les consideraba a estas como "especialistas" en dificultades de aprendizaje, de ahí que realizaran una labor más "terapéutica" que educativa con un alumnado específico (con NEE). Además, la profesora citada mencionó que los profesores de aula regular detentan un conocimiento más profundo de los contenidos de cada asignatura, por lo cual serían "expertos" en la enseñanza del lenguaje, las matemáticas, entre otras materias escolares.

De manera similar, las educadoras diferenciales entrevistadas en la fase exploratoria produjeron discursos tendientes a distinguir los saberes y los roles que detentaban en comparación con sus colegas de educación básica. Incluso consideraban que sus conocimientos tenían un grado mayor de especificidad sobre las dificultades del aprendizaje de los educandos y sobre su aporte en el aula regular y en el aula de recursos dirigidos a la enseñanza de todo el alumnado.

En el siguiente fragmento - rescatado de una entrevista realizada en la fase exploratoria a una educadora diferencial — se refleja lo aseverado con anterioridad:

A ver, las educadoras diferenciales somos las especialistas en las dificultades de aprendizaje en niños que presentan TEL (trastorno específico del lenguaje), TEA (trastorno específico del aprendizaje), DIL (déficit intelectual limítrofe) y autismo, pero lo más importante, es cómo ayudar a los niños que más les cuesta y en general a todos los niños, porque generalmente la gente cree que solo trabajamos con niños con necesidades educativas especiales. Entonces, nosotras completamos el trabajo que hace un profesor o una profesora en la sala de clases, porque nosotras tenemos formas más didácticas de cómo enseñar, ya que la mayor cantidad de problemas de aprendizaje son problemas de cómo los profesores de básica enseñan (15 de abril de 2020, entrevista 27). 


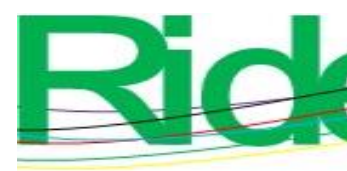

Revista Iberoamericana para la
Investigación y el Desarrollo Educativo
ISSN $2007-7467$

Para el caso de la educadora diferencial citada, se replica el discurso que las posiciona como las "especialistas" en dificultades del aprendizaje y del trabajo con niños que han sido catalogados con NEE, distinguiéndose por medio de un lenguaje técnico relacionado con las etiquetas diagnósticas con las que se nombran a los estudiantes (TEL, TEA, DIL, etc.), como también por el conocimiento de las políticas en materia de inclusión. Este lenguaje técnico vinculado con su formación inicial — en el que confluye una terminología psicopedagógica y educativa - les confiere un estatus profesional de una "especialista" que les provee un posicionamiento distinto al de los profesores de primaria. Al mismo tiempo, existía una presunción en el discurso de las maestras de educación especial que vinculaba un supuesto origen de los problemas de aprendizaje de los educandos con inadecuadas metodologías para enseñar, aplicadas por los docentes de educación básica.

Un matiz diferente a los discursos presentados se evidenció en las declaraciones que emitió una de las parejas correspondientes a los casos de estudio. Esta pareja diferenció sus saberes y roles de manera más patente, predominando narrativas que resultaron ser más taxativas relacionadas con los conocimientos y el estatus que se atribuían en el trabajo pedagógico cotidiano. De esta forma se refirieron la educadora Sofía y el profesor Raúl, respectivamente:

Mira, las educadoras somos las encargadas y especialistas en la inclusión y en las dificultades de aprendizaje de los estudiantes. Entonces, nosotras evaluamos a los niños, mejoramos sus dificultades y, por sobre todo, ayudamos a los niños y a profesores a superar sus problemas. Porque la verdad sea dicha, la mayoría de las dificultades que tienen los niños para aprender es porque los profesores de básica no saben cómo enseñar bien, o están ocupando malas metodologías o no tienen paciencia o tratan a los niños como tontitos (7 de abril de 2018, entrevista educadora Sofía).

Los profesores siempre hemos sido los expertos en la educación, en la enseñanza, en las asignaturas y en el cómo enseñar. Yo soy licenciado en Literatura y después fui profesor de escuela y somos expertos en lo que sabemos, en mi caso en el lenguaje y la literatura. Las educadoras diferenciales son especialistas en los niños que tienen problemas serios, en los que necesitan aprender en otro espacio, porque no tienen la capacidad de aprender como los demás compañeros, entonces, ellas se encargan de esos problemas en el aula de recursos (28 de marzo de 2018, entrevista profesor Raúl). 


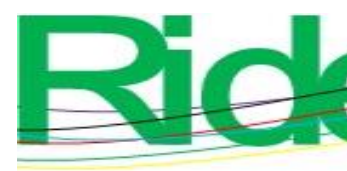

Revista Iberoamericana para la
Investigación y el Desarrollo Educativo
ISSN $2007-7467$

En ambos fragmentos se da cuenta de discursos que tienden a diferenciar de manera más patente los saberes y el estatus de cada uno de los entrevistados en la escuela municipal en cuestión. Con el fin de ofrecer mayor conocimiento de contexto en cuanto a los discursos emitidos, durante el trabajo de campo en este centro escolar se evidenció con frecuencia una relación tensa, de distanciamiento y problemas de comunicación y de coordinación en el trabajo pedagógico entre estos docentes. En el caso de la educadora Sofía, ella apunta a una especificidad de su trabajo relacionado con la inclusión, las dificultades de aprendizaje y la enseñanza. Igualmente, señala que los problemas para aprender del alumnado se debían al trabajo pedagógico de los profesores de aula, quienes no estaban desarrollando procesos educativos adecuados en términos metodológicos o didácticos. Para el docente Raúl, en cambio, los profesores de aula serían los expertos en la enseñanza de ciertas asignaturas dirigidas a los estudiantes que no tenían problemas; de esta forma, se transfiere la responsabilidad a la educadora diferencial de aquellos niños con dificultades, los que deberían —a su juicio - ser educados en otro espacio, como lo es el aula de recursos ${ }^{1}$. Así se establece una distinción clara entre el profesor de sala de clases que educa a niños y niñas "normales" y la pedagoga de educación especial que se encarga de la educación de los educandos “anormales", entendiendo esta denominación desde una perspectiva foucaultiana.

En síntesis, en los discursos expuestos con anterioridad se expresa una diferenciación en los saberes y el estatus que estos confieren a cada profesional de la educación. En el caso de los docentes de educación básica, estos tienden a posicionarse como los “expertos” en la enseñanza de determinadas asignaturas y de los contenidos específicos de cada una de ellas y con expertiz en el desarrollo de aprendizajes en todos los alumnos utilizando estratégicamente a su favor el saber-poder como especialista en la transposición didáctica aludiendo que su trabajo estaba vinculado a un ámbito más pedagógico que terapéutico. En tanto, las educadoras diferenciales entrevistadas se situaron como las "especialistas" en la inclusión, en las dificultades del aprendizaje y también de la enseñanza. En este sentido, relevaron el hecho de ejercer un saber-poder especializado en el trabajo con estudiantes con NEE y con aquellos educandos que no presentaban dificultades. Al mismo tiempo, estas docentes aludieron con frecuencia que los problemas para aprender del alumnado estarían situados en el aula regular, como efecto de metodologías inadecuadas o de derivación de los

\footnotetext{
${ }^{1}$ El aula de recursos es un espacio físico, como una sala de clases, dentro de los establecimientos educativos en donde las educadoras diferenciales trabajan con grupos reducidos de alumnos con NEE.
} 


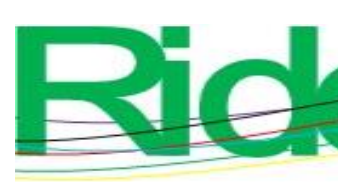

Revista Iberoamericana para la Investigación y el Desarrollo Educativo ISSN $2007-7467$

estudiantes con problemas hacia ellas, asumiendo hasta cierto punto que el "déficit" o la dificultad para aprender no se situaba en el sujeto escolar, sino en quien enseñaba en el aula regular. A su vez, estas pedagogas se validaban frente a sus colegas de educación básica a través del uso de un lenguaje biomédico o psicopedagógico, relacionado con las etiquetas diagnósticas del alumnado con NEE y en los discursos provenientes de las políticas de inclusión más contemporáneas.

En este punto, es relevante declarar que si bien tanto los profesores de educación básica como las educadoras diferenciales buscaban en las entrevistas distinguir sus roles y lenguajes, es también importante asumir que la diferencia que intentaban construir tenía relación con la formación inicial de cada uno y, principalmente, con los espacios en los que cada profesional se ha desempeñado tradicionalmente: el aula regular y el aula de recursos. Este último aspecto será analizado con mayor profundidad en el siguiente apartado.

\section{La disputa por el espacio y la autoridad en el aula regular}

Durante la fase de profundización se pudieron constatar diferentes formas en que el poder fue ejercido por parte de los profesores de educación básica hacia las educadoras diferenciales, y viceversa. De manera más específica, se identificaron por un lado situaciones de exclusión de la docente de educación especial y de los estudiantes con NEE dentro del aula regular, pedagogas que replicaban el trabajo que desempeñaban en el aula de recursos al interior de la sala de clases, aspecto evidenciado con frecuencia durante la coinstrucción en la pareja constituida por Sofía y Raúl. Por otra parte, se observó un desplazamiento del profesor de educación básica a una posición de ayudante de la educadora diferencial en el trabajo de coplanificación y coevaluación, para el caso de la dupla conformada por los pedagogos José y Patricia.

En el primer caso, el profesor de lenguaje, Raúl, durante una clase en el séptimo grado, impidió que la educadora diferencial Sofía desarrollara la coinstrucción (planificada en conjunto con anterioridad), por lo que esta docente fue desplazada a un rincón de la sala de clases, donde solo interactuó y apoyó pedagógicamente a los dos estudiantes con NEE, con los que desarrolló un trabajo distinto al que realizó el docente Raúl con el resto de la clase durante la sesión observada. Esa situación fue registrada en la siguiente nota de campo: 


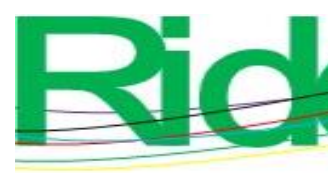

Revista Iberoamericana para la Investigación y el Desarrollo Educativo ISSN $2007-7467$

Mientras el profesor Raúl abre la presentación en formato PPT para proyectarla, le indica con el dedo (sin hablar) a Sofía, la parte de atrás de la sala.

La educadora diferencial se acerca a hablar con él, pero este no emite ninguna palabra y le indica a esta la parte de atrás de la sala de clases nuevamente con el dedo.

Sofía se traslada al final de la sala al lado donde estaba sentado el investigador. Habla con dos niños con NEE y les dice que se sienten junto a ella. Los alumnos asienten e inmediatamente se acercan a ella.

El profesor de lenguaje sigue impartiendo la clase al resto del curso y la educadora diferencial Sofía abre una carpeta en la que llevaba unas guías preparadas para estos dos alumnos y realizan actividades distintas a las que realizó el docente Raúl (2 de mayo de 2018, nota de campo).

En este episodio se puede observar cómo la educadora diferencial —encargada de la inclusión y denominada como "la especialista" por sus colegas de educación básica- fue desplazada no solo del trabajo de coenseñanza (específicamente de construcción), sino que también fue excluida junto a los dos alumnos con NEE de la participación en la dinámica de la clase y del mismo currículo, tal y como las políticas en esta materia lo mandatan. En este sentido, el trabajo que realizaba Sofía en el aula de recursos se estaría replicando en el aula regular, y de esta forma la docente de educación especial estaría aplicando su saber-poder en ese espacio reducido, tal como lo haría en el aula de recursos. Además, se debe declarar que en la sesión previa de coplanificación entre ambos pedagogos involucrados la situación observada fue similar, ya que la educadora con frecuencia se restringía solo a brindar apoyo para la construcción de la guía y material didáctico para diversificar la enseñanza, por lo que existía un diálogo muy limitado entre ambos profesores, centrado principalmente en sugerencias de Sofía, que eran rebatidas u omitidas por el docente Raúl.

La figura 1 ilustra la forma en que la educadora Sofía fue descartada en el episodio presentado con anterioridad. 

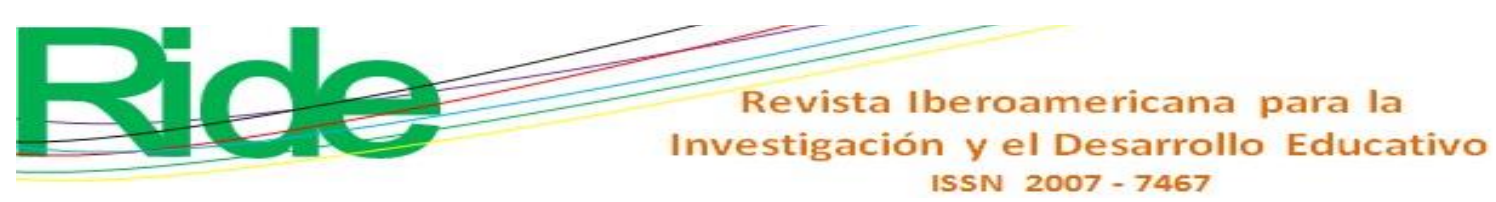

Figura 1. Sala de clases de séptimo grado

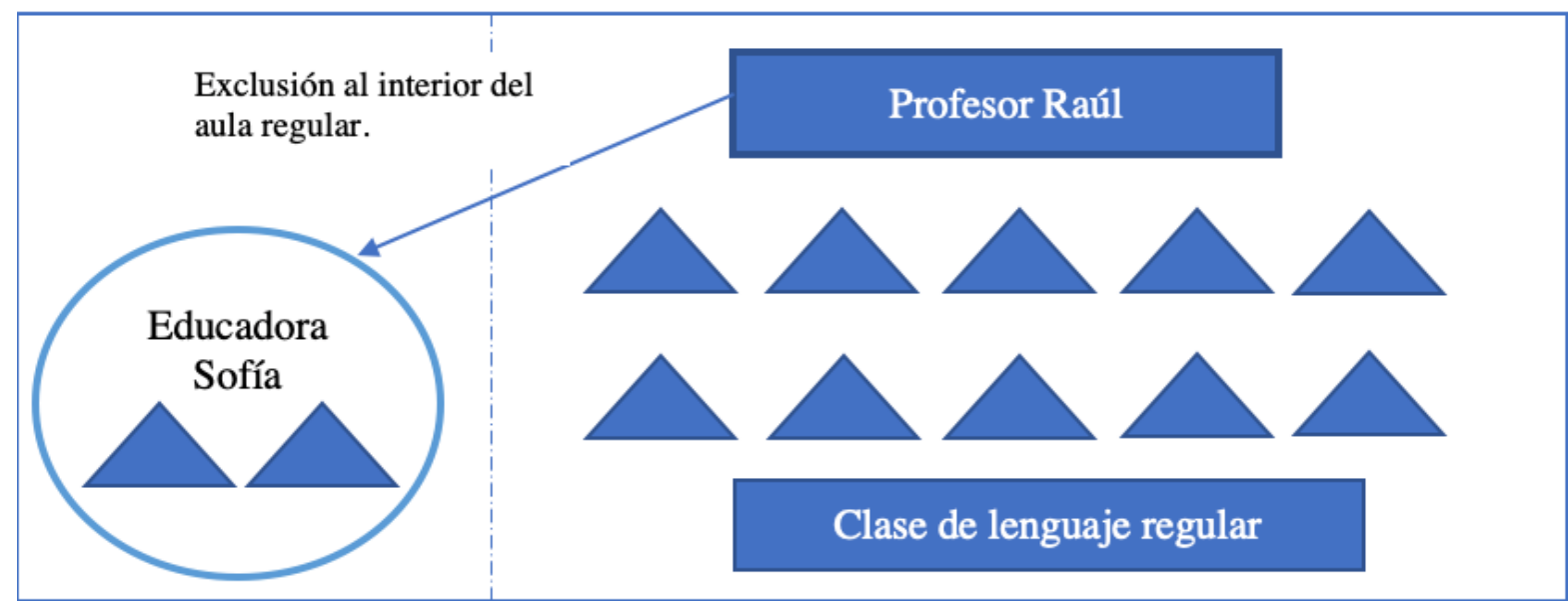

Fuente: Elaboración propia

Otro de los episodios observados en la interacción entre el profesor Raúl y la educadora Sofía - y que demostró una disputa de poder y autoridad dentro del aula regular en situaciones en las que se intentaba llevar a cabo la coenseñanza - se produjo al terminar una de las sesiones de lenguaje, cuando la jefa de UTP (unidad técnico-pedagógica) ingresó al aula regular (ya sin alumnos). En ese momento se sostuvo el siguiente diálogo, donde el docente Raúl indicó que Sofía debería estar en el aula de recursos con "sus niños", y no en la sala de clases:

Educadora Sofía (dirigiéndose a la jefa de UTP): Es cierto, yo no soy profesora de lenguaje y quizás no tengo la capacidad para enseñar todos los contenidos de lenguaje, pero yo tengo toda la voluntad de aprender y apoyar al colega aquí con todos los niños.

Profesor Raúl (dirigiéndose a la jefa de UTP): Ya, pero insisto, ella es la especialista, ella está a cargo solo de los estudiantes con problemas. ¿Por qué no es como antes donde la educadora sacaba a sus niños de la sala de clases? Ahora ella igual se debe sentir incómoda al trabajar con sus niños acá en la sala.

Educadora Sofía (dirigiéndose ahora al profesor): Mire, colega, yo no me siento incómoda por trabajar con mis niños, me siento incómoda porque usted me ningunea y ni siquiera toma en cuenta mis sugerencias para diversificar la enseñanza (13 de junio de 2018, nota de campo). 


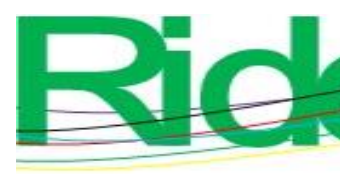

Revista Iberoamericana para la
Investigación y el Desarrollo Educativo
ISSN $2007-7467$

igualmente, dejó en evidencia el desconocimiento de este pedagogo sobre las dificultades del aprendizaje de algunos alumnos y de los mandatos de las últimas políticas que en materia de inclusión escolar se han promulgado en Chile.

La situación presentada con anterioridad se puede comprender de mejor forma al considerar que los profesores de educación básica de esta institución no contaban con el tiempo o las instancias de formación dentro del colegio para capacitarse en las políticas de inclusión escolar o en las NEE de los educandos, lo que se suma a la ausencia de acompañamiento pedagógico por parte de los directivos o de "expertos" en inclusión que guiarán o fortalecieran el trabajo pedagógico en conjunto entre las parejas de docentes. De esta forma lo manifestó el profesor José en una entrevista:

La verdad es que yo sigo las instrucciones de Patricia tal como ella me las dice, porque en la universidad no aprendimos casi nada de la inclusión o de las políticas y también de cosas como el DUA, que es algo nuevo. Lo único que hizo este colegio fue mandarnos a una capacitación para entender lo que es el DUA en el verano, un curso que hizo una ATE (asistencia técnica educativa) y la verdad no aprendimos casi nada, porque lo único que vimos fue la parte teórica del DUA y nada práctico de cómo hacer las clases con DUA o cómo trabajar con las colegas diferenciales (21 de octubre de 2018, entrevista 2, profesor José).

Con esta información se puede conocer con mayor detalle que políticas como el decreto 83 del año 2015 (donde se introdujo el DUA como estrategia de enseñanza para atender a la diversidad de necesidades educativas de todo el alumnado) no fueron debidamente comunicadas y posteriormente implementadas en este contexto, por lo que las educadoras diferenciales eran quienes lideraban el proceso de puesta en práctica de esta legislación. En consecuencia, eran las maestras de educación especial quienes lideraban las sesiones de coplanificación y coevaluación, aspecto que difería a la hora de desarrollar la coinstrucción en esta institución educativa.

Retomando el episodio presentado con anterioridad de co-planificación entre José y Patricia - donde la educadora era quien ejercía el saber-poder a su favor para que el docente de matemáticas diseñara las clases de acuerdo con sus recomendaciones_-, esta asimetría de poder se pudo revertir hasta cierto punto en el momento en que se desarrolló una clase de matemáticas en la que ambos pedagogos participaron en la construcción. En ese momento, José asumió la dirección de la sesión y Patricia actuó como un apoyo dentro de la sala de 


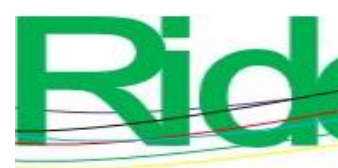

Revista Iberoamericana para la
Investigación y el Desarrollo Educativo
ISSN $2007-7467$

clases regular, atendiendo de manera particular las necesidades educativas de la niña que presentaba condición de autismo. A continuación, se registra la interacción entre ambos pedagogos en una clase de matemáticas en $6 .^{\circ}$ año de educación básica:

Profesor José (digiéndose a los educandos): Bien, niños, hoy trabajaremos con las fracciones y para esto vamos a pasarle una guía grupal de 4 integrantes, las que van a ir completando con la ayuda de sus compañeros. Con la profesora Patricia pasaremos puesto por puesto respondiendo todas las preguntas que tengan. Eso, ahora trabajen.

Educadora Patricia (dirigiéndose a los alumnos): Eso, yo también pasaré resolviendo dudas y preguntas que ustedes vayan teniendo, ya que la guía la hice con su profesor, así que si tienen preguntas no solo llamen a su profesor, sino que también me pueden llamar a mí. (Dicho eso, la educadora se quedó aproximadamente 20 minutos con el grupo donde se encontraba la estudiante con condición de autismo y solo respondió una pregunta de un grupo que se encontraba al lado de ella) (18 de junio de 2018, nota de campo).

Como se puede evidenciar, la distribución del poder en esta ocasión fue diferente a la observada en la sesión de coplanificación, ya que la asimetría de poder en esta situación se inclinó en favor del profesor de matemáticas, quien dirigió la sesión de clases. En contraste, la educadora Patricia se limitó a trabajar casi de forma exclusiva con el grupo de estudiantes donde se hallaba la alumna con una condición de autismo. Además, Patricia solo respondió una consulta que le realizó un grupo que se encontraba cerca de ella. A su vez, es preciso señalar que los educandos solo consultaban al profesor; es decir, omitían la presencia de la educadora en el aula.

En definitiva, fue en el aula regular - específicamente en la clase de matemáticasdonde se constató una dinámica distinta a la observada con frecuencia en las tareas de coplanificación, es decir, cuando la educadora ejercía a su favor el saber-poder como especialista. En otras palabras, ahora el profesor de matemáticas era quien tomaba el protagonismo de las clases, y la docente Patricia era solo una "ayudante" para apoyar especialmente a la niña con condición de autismo.

Asimismo, cabe destacar que la aplicación del DUA en este colegio en particular era responsabilidad de las educadoras diferenciales, de ahí que los profesores de aula regular se desligaran de esta tarea. Por ello, tanto en las sesiones de coplanificación y coevaluación, era 


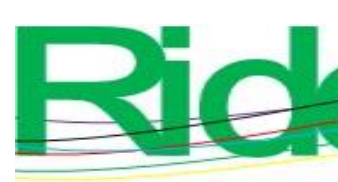

Revista Iberoamericana para la Investigación y el Desarrollo Educativo ISSN 2007 - 7467

Patricia quien dirigía y tomaba las decisiones pedagógicas más relevantes, lo que contrasta con las sesiones de coinstrucción en las que José impartía los contenidos y dirigía la clase.

\section{Discusión}

El objetivo de este estudio fue conocer las relaciones de poder presentes en tareas de coenseñanza de dos parejas de profesores de escuelas subvencionadas de la Región Metropolitana. En este proceso se detectaron dos categorías claves: en primer lugar, los saberes y el estatus del experto y de la especialista y, en segundo lugar, la disputa por la autoridad y el espacio en el aula regular.

Respecto a la primera categoría, se pudo constatar que el discurso por medio del cual los profesores de educación básica legitimaban su estatus como profesionales estaba relacionado con la figura de un "experto” en la enseñanza, específicamente, en la didáctica y en el conocimiento de ciertas asignaturas, lo que les conferían saberes curriculares que los distinguían de sus colegas educadoras diferenciales. En cambio, el saber-poder por medio del que las educadoras diferenciales se distinguían de los profesores de primaria estaba vinculado con su identidad y con el lenguaje relacionado con las etiquetas diagnósticas de los alumnos con NEE y de las políticas de inclusión, “jerga” más cercana al trabajo de un terapeuta o de un profesional de la salud que a la de un pedagogo.

En cuanto a la segunda categoría, se evidenció una disputa por el poder dentro de la sala de clases regular, donde ambos pedagogos debían desempeñarse desarrollando la coinstrucción. En el primer caso de estudio, Sofía fue excluida dentro del aula en las sesiones de lenguaje, por lo que fue omitida como una profesional que podía aportar en la enseñanza. De hecho, el profesor Raúl la desplazó hacia un rincón del espacio y la limitó a trabajar solo con los estudiantes que presentaban NEE. Igualmente, se constató que la educadora replicó las labores que realizaba en el aula de recursos en la sala de clases con los educandos etiquetados con problemas de aprendizaje. La situación expuesta con anterioridad es coherente con lo observado en los momentos de coplanificación y coevaluación, donde la educadora fue posicionada como una subalterna del profesor de aula. A esto se suman los problemas a nivel comunicacional y de coordinación de actividades entre esta pareja de docentes. 


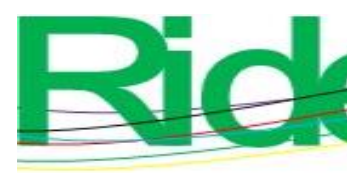

Revista Iberoamericana para la Investigación y el Desarrollo Educativo ISSN $2007-7467$

Por otra parte, en las relaciones de colaboración entre el profesor José y la educadora Patricia, el poder se distribuyó y se ejerció diferencialmente de acuerdo con las tareas de coenseñanza desarrolladas entre ambos. En las tareas de coplanificación y coevaluación se identificó que Patricia era quien se legitimaba por medio de su saber-poder en la elaboración de los planes de clases. En constraste, se evidenció que en tareas de coinstrucción en la sala de clases, el docente José era quien protagonizaba la sesión, tomaba la palabra, dirigía y monitoreaba el aprendizaje de los alumnos, al contrario de lo que sucedía con Patricia, quien se centraba a trabajar casi que exclusivamente con el grupo en el que se hallaba una niña con condición de autismo. En otras palabras, la relación de poder se invertía a favor del profesor.

Por lo anterior, se puede decir que en este estudio se refuerzan los hallazgos encontrados en otros trabajos desarrollados a nivel nacional relacionados con las dificultades para implementar la coenseñanza entre dos pedagogos que no comparten la misma formación inicial, que no cuentan con el tiempo y el espacio para coordinar el trabajo en conjunto y que tampoco son apoyados en el proceso de puesta en práctica de políticas que buscan favorecer la coenseñanza (Marfán et al., 2013; Figueroa-Céspedes et al., 2020; Urbina et al., 2017). Aunado a esto, se puede decir que el poder se distribuye dependiendo del saber-poder de cada profesional, es decir, según la formación inicial docente de cada uno y de su identidad, así como por el espacio o la tarea de coenseñanza desarrollada por los educadores en cada contexto escolar.

Esta investigación, además, aporta algunas luces de cómo problematizar las relaciones de poder cuando dos pedagogos trabajan juntos, ya que no solo se requiere de una formación en el trabajo colaborativo en los centros escolares, sino que también es indispensable que la escuela como institución favorezca espacios de trabajo conjunto y de reflexión para dotar de sentido la finalidad última del proceso educativo: el aprendizaje de todos los estudiantes, lo cual transciende los roles, funciones, lenguajes y jergas que cada profesional despliega en su trabajo pedagógico cotidiano.

Por último, los hallazgos de este estudio pueden ser empleados para comprender la vertiente productiva del poder, es decir, cómo en las tareas de coenseñanza las subjetividades de los docentes se ven transformadas en virtud de cómo el poder circula, fluye y es ejercido estrategicamente por cada uno de los educadores. En este estudio se da cuenta en una muestra acotada de docentes (dos parejas) la manera en que surge el antagonismo o la colaboración durante tareas de coenseñanza. Asimismo, se evidencia cómo las políticas implementadas bajo un esquema top-down afectan las micropolíticas de las escuelas y principalmente las 


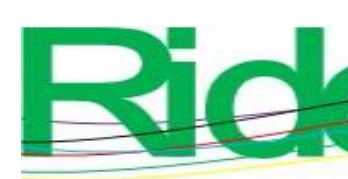

Revista Iberoamericana para la
Investigación y el Desarrollo Educativo ISSN $2007-7467$

subjetividades de docentes (Ball, 1997; Falabella, 2020), quienes deben convertirse en "empresarios de sí mismos" (Grinberg, 2008), gestionando los cambios que incorporan las políticas educativas en sus contextos de desempeño y responsabilizándose tanto de los deberes que mandatan las políticas, como de los resultados de la implementación de estas legislaciones. Esto, en definitiva, los tensiona porque se les transfiere la responsabilidad del éxito o del fracaso en iniciativas tan relevante como la coenseñanza y la inclusión escolar.

\section{Conclusiones}

La presente investigación da muestra de cómo el poder es ejercido y distribuido asimétricamente en la interacción de distintos profesionales de educación durante las tareas de coenseñanza. Específicamente, en Chile es relativamente nuevo el trabajo colaborativo entre profesores de educación básica y educadoras diferenciales, de ahí que existan aún muchos nudos críticos que deben ser analizados para crear futuros líneas de estudio; por ejemplo, las relaciones de género en tareas de coenseñanza, el papel de los directivos en la promoción de esta modalidad de trabajo pedagógico e incluso examinar las estructuras, micropolíticas y rutinas de trabajo en las instituciones educativas para establecer cuáles cambios son pertinentes para que la coenseñanza efectivamente suceda y favorezca el aprendizaje de los estudiantes.

En el caso del presente estudio, conocer con mayor detalle las relaciones de poder en las tareas de coenseñanza evidenció que el ejercicio del poder en la interacción entre pedagogos estuvo mediado por la identidad, la formación inicial docente (FID) y el saberpoder que cada profesional ejercía. En otras palabras, más que desarrollar un trabajo centrado en el aprendizaje de los alumnos, cada profesional buscaba legitimarse frente al otro a través de su expertiz; en consecuencia, se olvidaban de colocar sus conocimientos y formación al servicio de un aprendizaje que fuese accesible para todos los educandos. Asimismo, se pudo identificar que los espacios y los momentos de la coenseñanza fueron relevantes para distinguir cómo el poder se distribuía y circulaba entre ambos pedagogos.

Por otra parte, en cuanto a las limitantes detectadas en la investigación, se puede mencionar el tamaño de la muestra seleccionada; por ende, los resultados de este estudio no pueden ser extrapolados a todas las relaciones de poder entre pedagogos en tareas de coenseñanza en el país, ya que estas interacciones son dinámicas y varían de acuerdo con algunos factores que en esta investigación fueron evidenciados, tales como FID, contextos 


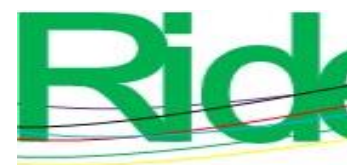

Revista Iberoamericana para la
Investigación y el Desarrollo Educativo
ISSN $2007-7467$

de desempeño, apoyo en la implementación de las políticas por parte de los directivos, entre otros.

Por tanto, se puede concluir que este estudio aporta algunas claves para mejorar la formación inicial docente (FID) tanto de educadoras diferenciales como de profesores y profesoras de educación general básica, específicamente en la preparación para el trabajo de coenseñanza. Asimismo, se podrían considerar políticas públicas en estas materias que consideren transformaciones en las instituciones educativas, las cuales deberían de brindar apoyos, recursos y tiempos necesarios para que la cultura de la colaboración pueda desarrollarse. De hecho, las legislaciones deben prever que en las escuelas también pueden surgir conflictos que de una u otra manera influirán en el éxito o fracaso de sus propósitos. En este sentido, los hallazgos de esta investigación proporcionan evidencia empírica respecto a la relevancia de generar cambios en la formación de pedagogos y en la formulación de políticas públicas en educación que fomenten apoyos integrales para que la inclusión escolar y el aprendizaje de todos los estudiantes — sin distinciones — sea una realidad, y no una promesa aún incumplida.

\section{Futuras líneas de investigación}

Se espera que se puedan proyectar estudios que consideren enfoques etnográficos para examinar en el trabajo cotidiano de educadoras diferenciales y profesores de educación básica, desde el prisma de las relaciones de poder vinculadas con el género de estos educadores, aspecto que fue tangencialmente tratado en esta investigación. Del mismo modo, se pueden extrapolar los resultados de este artículo, en el estudio de las micropolíticas de las escuelas en Chile, en las que se busca mejorar la calidad de la educación ofrecida empleando enfoques provenientes del Nuevo Management Público (NPM) y el accountability con altas consecuencias, esquemas bajo los que las escuelas y los actores educativos, se esfuerzan más en lograr cumplir con los estándares de aprendizaje exigidos por las agencias estatales y las familias, más que en promover actitudes, políticas y prácticas inclusivas, dejando a la justicia social y a la inclusión escolar como temáticas de segundo orden o como tareas pedagógicas optativas y sujetas a la "buena" voluntad de cada establecimiento educativo. 


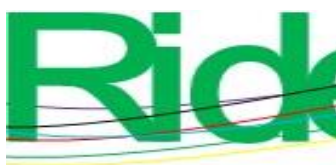

Revista Iberoamericana para la Investigación y el Desarrollo Educativo ISSN $2007-7467$

\section{Referencias}

Aliakbari, M. and Bazyar, A. (2012). Exploring the impact of parallel teaching on general language proficiency of EFL Learners. Journal of Pan-Pacific Association of Applied Linguistics, 16(1), 55-71.

Ball, S. (1997). Foucault y la educación. Disciplinas y saber. Madrid: Morata.

Cardona, M. (2002). Introducción a los métodos de investigación en educación. Madrid: Editorial EOS.

Cherryholmes, C. (1999). Poder y crítica. Investigaciones postestructurales en educación. Barcelona: Pomedores- Corredores.

Conderman, G. and Hedin, L. (2012). Purposeful assessment practices for coteachers. Teaching Exceptional Children, 44(4), 19-27.

Cook, L. and Friend, M. (1995). Co-teaching: guidelines for creating effective practices. Focus on Excepcional Children, 28, 1-25.

Cramer, E., Liston, A., Nervin, A. and Thousand, J. (2010). Co-teaching in Urban Secondary School. districts to Meet the Needs of all Teachers and Learners: Implications for Teacher Education Reform. International Journal of Whole Schooling, 6(2), 59-76.

Creswell, J. (2007). Qualitative inquire and research design: Choosing among five approaches. Thousand Oacks: SAGE.

Da Silva, T. (2001). Espacios de identidad: nuevas visiones sobre el currículum. Madrid: Octaedro.

Decreto 170 (2009). Decreto con toma de razón $N^{\circ}$ 170. Fija normas para determinar los alumnos con necesidades educativas especiales que serán beneficiarios de las subvenciones para educación especial. Santiago: Chile.

Falabella, A. (2020). The ethics of competition: accountability policy enactment in Chilean schools' everyday life. Journal of Education Policy, 35 (1), 23-45.

Figueroa-Céspedes, I., Sepúlveda Guajardo, G., Soto Cárcamo, J. y Yáñez-Urbina, C. (2020). Coenseñanza entre docentes de educación general básica y educadoras diferenciales: incidentes críticos de la práctica colaborativa en proyectos de integración educativa. Pensamiento Educativo. Revista de Investigación Educacional Latinoamericana, 57(1), 1-15.

Foucault, M. (2006). Seguridad, territorio y población. Curso en el College de France(19771978). Buenos Aires, Argentina: Siglo XXI Editores. 

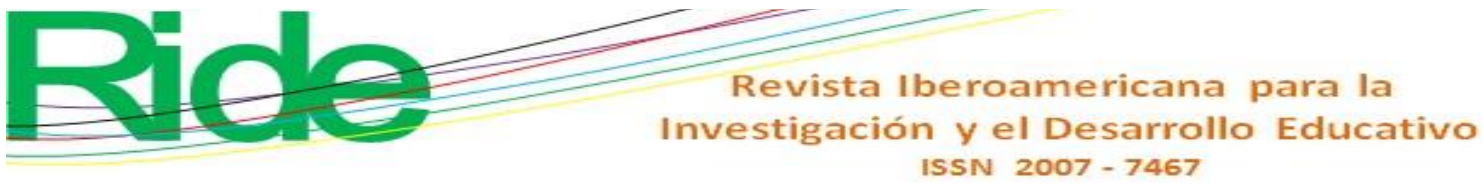

Strauss A. and Corbin, J. (1998). Basics of qualitative research: grounded Theory procedures and techniques. Newbury Park: SAGE.

Strogilos, V. and Stefanidis, A. (2015). Contextual antecedents of co-teaching efficacy: Their influence on students with disabilities' learning progress, social participation and behaviour improvement. Teaching and Teacher Education, 47, 218-229.

Suárez-Díaz, G. (2016). Co-enseñanza: concepciones y prácticas en profesores de una Facultad de Educación en Perú. Revista Electrónica de Investigación Educativa, $18(1), 166-182$.

Tójar, J. (2006). Investigación cualitativa. Comprender y actuar. Madrid: La Muralla.

Urbina, C., Basualto, P., Durán, C. y Miranda, P. (2017). Prácticas de co-docencia: el caso de una dupla en el marco del Programa de Integración Escolar en Chile. Estudios Pedagógicos, 43(2), 355-374.

Villa, R., Thousand, J. and Nevin, A. (2008). A Guide to Co-teaching. Practical Tips for Facilitating Student Learning. Thousand Oaks: Corwin Press.

Yin, R. (2010). Qualitative Research from start to finish. London: The Guilford Press. 